\title{
A Retrospective Comparative Study on Median Time to Sputum Culture Conversion in Multi-Drug Resistant Pulmonary Tuberculosis Patients in Pastoral and Non-Pastoral Settings in Southeast Oromia, Ethiopia
}

\author{
Abebe Megerso $\mathbb{D D}^{1}$ \\ Negusie Deyessa ${ }^{2}$ \\ Godana Jarso ${ }^{3}$ \\ Alemayehu Worku (iD) ${ }^{2}$ \\ 'Department of Public Health, Adama \\ Hospital Medical College, Adama, \\ Oromia, Ethiopia; ${ }^{2}$ Department of \\ Preventive Medicine, School of Public \\ Health, Addis Ababa University, Addis \\ Ababa, Ethiopia; ${ }^{3}$ Department of \\ Medicine, Adama Hospital Medical \\ College, Adama, Oromia, Ethiopia
}

Purpose: Sputum culture conversion to negative is an indicator of good interim treatment outcome. Pastoralist community has lesser access to healthcare services. This study aimed to compare the time to culture conversion (TTSCC) between the pastoral and non-pastoral settings and identify its determinants among drug-resistant pulmonary TB patients.

Patients and Methods: Four hundred forty-seven drug-resistant pulmonary TB patients were included from selected hospitals of southeastern Oromia, Ethiopia. Kaplan-Meier model using the Log rank test was fit to compute and compare median TTSCC between study participants from the pastoral and non-pastoral settings. The Cox proportional hazard model was fit to identify factors associated with the TTSCC. Adjusted hazard ratio (AHR) with a 95\% confidence interval (CI) was used to report the strength of association. Statistical significance was declared at $\mathrm{p}<0.05$.

Results: The study participants' median age (interquartile range) was 29 (24-36) years. The overall median TTSCC among the current study participants was 67 (95\% CI, 64-70) days. It was significantly different for patients from the pastoral and non-pastoral settings ( $p$-value $<0.001)$. The median TTSCC for patients from pastoral and non-pastoral settings was 101 (95\% CI, 81-121) and 63 (95\% CI, 61-64) days. To mention few determinants, patients from the pastoral setting had a $69 \%$ lower chance $[\mathrm{HR}=0.31(95 \%$ CI $0.24-0.41)]$ of shorter TTSCC than patients from the non-pastoral setting. Furthermore, patients with baseline body mass index greater than $18.5 \mathrm{Kg} / \mathrm{M}^{2}$ had a $35 \%$ higher chance of shorter TTSCC $[\mathrm{HR}=1.35$ (95\% CI 1.07-1.71)] compared to their counterparts.

Conclusion: The median TTSCC among the study participants from pastoral was longer than those from non-pastoral setting. The pastoral setting, under-nutrition, previous exposure to anti-TB drugs and drug regimen categories were among the notable determinants of the TTSCC among our study participants. Hence, due attention should be given to patients with these determinants during the treatment.

Keywords: Ethiopia, multi-drug tuberculosis, Oromia, pastoral setting, time to sputum culture conversion, treatment outcome

\section{Plain Language Summary}

Tuberculosis (TB) remained one of the top ten killer diseases in the world. It is the first cause of death due to a single infectious disease in the world in 2019. Development of drugresistant strains of the bacterium that causes TB threatened the success gained in controlling
Tel +25। 911044525

Email abemegerso@gmail.com; abebe. megerso@aau.edu.et 
the disease. These drug-resistant bacterial strains cause a form of the disease, which is challenging to treat and requires more toxic medications taken over longer period than those taken for the non drug resistant TB. Sputum culture conversion to negative during treatment of patients with TB of the lungs is good news to the patients and the community.

This study aimed to determine the average time for sputum culture conversion among drug-resistant pulmonary TB patients and to identify issues determining this time. To attain these objectives, authors analyzed records of 447 patients obtained from hospitals found in southeastern Oromia region of Ethiopia. Applying appropriate data analysis methods, we came up with useful evidences, which are described as follows.

The average time to sputum culture conversion among the study participants was longer than two months in overall patients and three months in patients from pastoral setting. This long time means that patients continue shading infective resistant bacteria for months, even after starting the treatment. This shading, in turn, results in sustained transmission of the infection in the community. The pastoral setting, educational status, undernutrition, previous exposure to anti TB drugs, and drug regimen categories were among our study participants' significant factors contributing to the average time.

Therefore, we believe that disseminating these results using possible means will inform concerned stakeholders, including community members, to pay due attention to patients with the determinants of the time to sputum culture conversion during the treatment and beyond to prevent and control the disease in the reality.

\section{Introduction}

Drug-resistant tuberculosis (DR TB) is an infectious disease caused by anti-tuberculosis drug resistant strains of Mycobacterium Tuberculosis bacteria. ${ }^{1}$ Drug-resistant TB has different classifications. These include monoresistance when the bacterium is resistant to only one antiTB drug; poly resistance when the bacterium is resistant to more than one anti-TB drugs but not for isoniazid and rifampicin at the same time. If the bacterium is resistant to both isoniazid and rifampicin, it becomes MDR TB. Furthermore, the bacterium can be resistant to any of the fluoroquinolones and at least one of the three second-line injectable drugs (capreomycin, kanamycin and amikacin) in addition to multi-drug resistance. In that case, the disease caused by such a bacterium is labeled as extensively drug-resistant TB (XDR-TB). ${ }^{1-3}$ Development of DR TB became a challenge in the fight against the disease in the world in general and in TB high burden and developing countries in particular. $^{2}$ It is difficult to treat DR TB than drug-susceptible TB and presents a significant challenge for patients, healthcare workers and healthcare services. ${ }^{3}$ The increase in the burden and incidence of DR TB threatens global progress towards the targets set by the End TB Strategy of the World Health Organization (WHO). ${ }^{4,5}$

Tuberculosis remained one of the top 10 causes of death worldwide and the leading cause of death from a single infectious agent. In 2019, about 10 million incidents of all forms of TB cases were estimated, and 7.1 million were reported. ${ }^{2}$ In the same year, over 500,000 incident MDR-TB or rifampicin-resistant TB (RR-TB) was estimated globally, of which slightly higher than 206,000 cases were reported. ${ }^{2}$ Based on the estimated absolute number of cases, Ethiopia is among the top 20 high TB, TB/HIV and MDR-TB burden countries. ${ }^{2}$ Estimated rate of TB incidence in Ethiopia in 2019 was 140 cases per 100,000 population, and the estimated number of incident cases was 157,000 with an uncertainty range between 110,000 and 211,000 cases. $^{2}$ In Ethiopia, $0.71 \%$ of new and $12 \%$ of previously treated TB cases were estimated to be infected with MDR/RR-TB bacterium. In 2019, estimated incident cases of TB were 1400 in the country, of which $579(41.4 \%)$ were reported in the year. ${ }^{2,3}$

Guidelines for diagnosis and treatment of MDR/RR-TB are progressively changing based on updated recommendations. Patients included in this study were diagnosed using Xpert MTB/RIF assay, a rapid molecular test to identify the development of resistance to rifampicin. ${ }^{6,7}$ Once RR-TB is specified, the patient starts the MDR/ RR-TB treatment, and the sample is sent for other drugs susceptibility tests such as first-line line-probe assay and baseline culture. ${ }^{1,6}$ Treatment of MDR/RR-TB is different from drug-susceptible TB cases in terms of the drug regimen composition, length of the treatment duration and the drug toxicity. ${ }^{8}$ Timely conversion of sputum culture among pulmonary TB patients is good news for the patients and essential for the community. This important indicator of treatment outcome is affected by different factors, including various socio-economic determinants. ${ }^{9-12}$ Sputum culture is said to be converted to negative when two consecutive cultures, taken at least 30 days apart, are found to be negative and no reversion to positive is reported. In such a case, the specimen collection date of the first negative culture is used as the conversion date. $^{6}$

Evidence indicates that $\mathrm{TB}$ treatment outcome is affected by social determinants. ${ }^{13-15}$ Pastoralist community setting is distinct from the non-pastoral community 
setting in terms of livelihood and access to health care. ${ }^{16}$ In this setting, the community moves from place to place in search of pasture on a water for livestock; hence access to healthcare services is limited. In the pastoralist community setting, TB patients face multifaceted challenges that can affect their treatment outcome. ${ }^{17}$ Nonetheless, evidence addressing disparities in TB treatment outcome in general and in the time to sputum culture conversion in particular among TB patients from pastoral and nonpastoral community setting is at least not readily available. Therefore, this study was planned to compare the TTSCC between the two livelihood settings (pastoral and nonpastoral) and to identify determinants of TTSCC among the study participants in the Oromia regional state of Ethiopia.

\section{Materials and Methods Study Area, Period, Design and Population}

The study was conducted in selected health facilities in the southeastern part of the Oromia regional state in Ethiopia. The facilities were purposefully selected from two distinct livelihood settings, namely the pastoral and non-pastoral settings. A retrospective cohort study design, involving MDR/RR-TB patients enrolled in the selected health facilities over four years (January, 2015 to December, 2019) was used. Sample size was calculated using a computer program designed to determine the sample size for survival analysis, the power and sample size calculator version 3.1.2. ${ }^{18,19}$

The sample size was calculated based on two parameters, time to event (sputum culture conversion) and estimated hazard ratio (HR). Common assumptions of confidence level and power, such as $95 \%$ confidence interval for the HR and $80 \%$ power, were considered for the sample size calculation. The ratio of patients from the non-pastoral community to those from the pastoral community was 1.5 . To calculate sample size using a time-toevent parameter, we considered an accrual interval of 48 months and additional follow-up after the accrual interval of two months. Previous study indicates that the median survival time on the control treatment, non-pastoral setting, in this case, was two months. ${ }^{20}$ Considering the expected delay of three weeks among the pastoral setting patients in time to sputum culture conversion compared to those from the non-pastoral setting, 134 and 201 (a total of 435) patients were required from the pastoral and non- pastoral settings, respectively. As we used existing data, we included all available records that met the inclusion criteria from both locations, increasing the sample size to 447 (177 from the pastoral and 270 from the non-pastoral setting).

\section{Inclusion and Exclusion Criteria}

Inclusion criteria: People with sputum culture positive DR TB at the beginning of the treatment and 15 years or older were included.

Exclusion criteria: Study participants diagnosed with pulmonary DR TB who meet the above inclusion criteria but reside in zonal towns of the pastoral setting were excluded. These patients were excluded because their inclusion may mask the effect of pastoral scenes on the TTSCC. Furthermore, patients enrolled before the past four years were excluded because MDR/RR-TB management was limited to the country's center.

\section{Study Variables (Dependent and Independent Variables)}

Time to sputum culture conversion was the dependent variable. Socio-demographic variables and clinical characteristics are independent variables. Patients' livelihood setting as dichotomized into the pastoral and non-pastoral setting is the main exposure variable.

\section{Data Collection Tool and Procedure}

The data extraction tool was designed to include all variables indicated in the national MDR/RR-TB management guidelines. ${ }^{3,6}$ The tool was used to extract data from patients' logbooks and clinical monitoring charts by trained data collectors.

\section{Data Quality Assurance}

The principal investigator closely supervised the data collectors during data collection. Filled data extraction tools were checked for consistency and against original patient records, logbook and patient monitoring charts. Data was entered into Epi-Info version seven and exported to statistical package and service solutions (SPSS) version 26 computer application software for analysis. ${ }^{21,22}$

\section{Data Analysis}

Data were analyzed using SPSS computer application software. Descriptive analysis was made to characterize the study participants. The Kaplan-Meier model and the Log 
rank test were fit to compare median TTSCC between study participants from the pastoral and non-pastoral settings. The Cox proportional hazard model was fit to control the effect of confounders. First, the binary Cox proportional hazard regression model was fit to assess the association of individual variables with the TTSCC. Then, all variables with a p-value less than 0.25 in the bivariate analysis were selected for the final or multivariable Cox proportional hazard regression model.

Proportionality of hazard was checked using the log minus the graphical log method. We assumed independence of survival time between the compared groups of study participants and no change in the independent variables for most variables. The assumption of nondifferential censoring between compared groups was checked by comparing the censored and non-censored observations.

The hazard ratio with its $95 \%$ confidence interval (CI) was used to report the strength of association between the independent and dependent variables. We used a P-value of less than 0.05 to report a statistically significant association between the dependent and independent variables.

\section{Results}

Medical records of 447 MDR/RR-TB patients, of which 177 (39.6\%) were from pastoral settings, were included in this analysis. The study participants' median age (interquartile range-IQR) was 29 (24-36) years. Males accounted for about 59\% and 264 (59.1\%) of the study participants were married. Majority of the study participants, 309 (69.2\%), had no formal education or attended just primary school. Furthermore, 187 (41.8\%) of the study participants were farmers or housewives.

With respect to clinical characteristics of the study participants, majority of them, $382(85.5 \%)$, were RR TB patients, which may be due to the inaccessibility of drug susceptibility tests for other drugs. A significant proportion of patients, $298(66.6 \%)$, had a previous treatment history with first-line anti TB drugs and 159 (37.1\%) of the study participants had BMI of less than $18.5 \mathrm{Kg} /$ Squ.M.

Of the total study participants, $406(90.8 \%)$ had their sputum culture converted to negative during the period included in this study. Among those who had their sputum culture converted 117 (28.8\%), 159 (39.2\%), and 57 $(12.8 \%)$ converted in the first two months, third month and fourth month, respectively. Nearly $10 \%$ (40 patients) remained sputum culture-positive beyond six months of treatment. The overall median TTSCC among the study participants was 67 (95\% CI, 64-70) days. The TTSCC was significantly different for patients from the pastoral and non-pastoral settings (Log rank test p-value was $<0.001)$. The median times to sputum culture conversion for the pastoral and non-pastoral setting were 101 (95\% CI, 81-121) and 63 (95\% CI, 61-64) days, respectively (Figure 1).

Of the variables categorized under the sociodemographic characteristics, educational status, occupation and livelihood setting came out to be independently associated with TTSCC adjusted for all other variables in the table (Table 1). Patients from the pastoral setting had $69 \%$ lower hazard $[\mathrm{HR}=0.31$ (95\% CI $0.24-0.41)]$ of timely sputum conversion to negative compared to patients from the non-pastoral community setting.

From the variables categorized under clinical characteristics of the study participants and modeled together as shown above (Table 2), baseline body mass index (BMI) of the patients, treatment registration group and composition of drug regimens given during the intensive and continuation phase of the treatment were independent determinants of TTSCC. Patients with a good baseline BMI, $18.5 \mathrm{Kg} / \mathrm{M} 2$, had a $35 \%$ higher hazard of timely sputum culture conversion; $[\mathrm{HR}=1.35(95 \%$ CI $1.07-$ 1.71)] compared to those with a lower baseline BMI (Table 2).

\section{Discussion}

In this study, two sets of essential evidences were produced. The first result is the median TTSCC to negative, for the overall study participants and as disaggregated by setting, among MDR/RR pulmonary TB patients. The second is identification of determinants of the TTSCC. The median TTSCC to negative among the study participants was more than two months; that is 67 days. Various previous studies reported different median TTSCC. Our result was consistent with a median time reported by a couple of studies. ${ }^{23,24}$ Yet, some of the previous studies have reported shorter median TTSCC. ${ }^{12,25-27}$ Studies reporting shorter median time are from better off settings compared to our study setting and this difference in setting may explain the observed difference in the median time.There are also many studies reporting longer TTSCC than the time reported in this study. ${ }^{11,20,28}$ These differences in the 


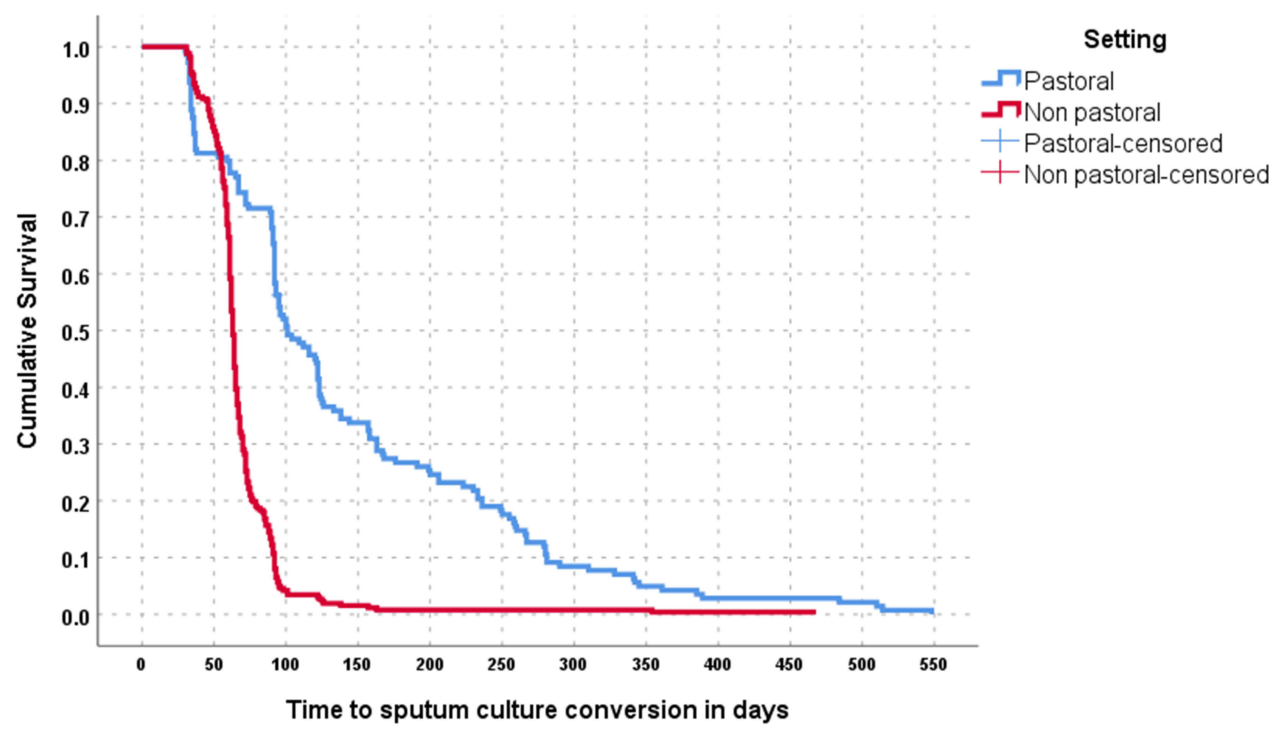

Figure I Comparison of the median time (days) to sputum culture between patients from the pastoral and non-pastoral setting, southeastern Oromia, Ethiopia, 2021.

TTSCC could be attributed to various determinants that may vary from a setting to another. These variations support the significant difference in the TTSCC in different livelihood settings of the same region we reported in this study.

Despite the variability in TTSCC, the implication of long time with unconverted sputum in pulmonary TB patients is more important given the treatment approach in Ethiopia. In Ethiopia, patients with MDR/RR-TB start anti TB treatment in treatment initiation centers located in major hospitals, and the treatment follows up and refilling is given in peripheral health facilities as outpatient services in treatment follow-up centers. That means patients live in the community. If the bacterium is not cleared from the patient, transmission of resistant Mycobacterium strains continues in the community. As depicted in this study, TTSCC among the patients from the pastoral community settings, was significantly longer than patients from the agrarian setting. In the pastoral community setting, living conditions and access to healthcare facilities are poor compared to the nonpastoral setting ${ }^{16,17}$ The difference in livelihood condition and access to health care can be the reason for longer TTSCT among patients from the former setting. This implies that infection transmission is maintained in such a community, which can negatively affect achievement the goals set for the TB prevention and control program.
The second set of evidence produced in this study was determinants of the time to culture conversion. Low BMI, treatment registration group and regimen categories during the intensive and continuation phase were identified as independent determinants of the TTSCC. Low BMI (less than $18.5 \mathrm{~kg} / \mathrm{sq} . \mathrm{m}$ ) was associated with prolonged TTSCC. This result is consistent with reports from several studies. Many studies reported results which were similar in the direction of association with our study result except slight differences in the strength of the association. ${ }^{20,23,28-30}$ Under-nutrition is more common in the pastoral community, and this could have contributed to the significant difference in the TTSCC between the pastoral and non-pastoral settings in this study. Patients who had a history of first-line anti-TB treatment failure had shorter TTSCC time in this study than those categorized as new to anti TB treatment. Consistent with this, patients with previous treatment history with second-line anti TB drugs showed shorter TTSCC, although not statistically significant in the final model. Other researchers have also reported faster time to the sputum culture conversion among those patients, ${ }^{27}$ Two possible justifications can be mentioned for these results. First, patients with history of treatment failure and diagnosed with resistant bacterial strain might have learned from their previous mistakes and have adhered to the current treatment. Second, the reference category might not be new to the treatment, particularly in the pastoral 
Table I Association of the Socio-Demographic Characteristics with the TTSCC Among MDR/RR-TB Patients in Southeastern Oromia, Ethiopia, 2021

\begin{tabular}{|c|c|c|c|c|}
\hline Variables & Number & Percent & CHR (95\% Cl) & AHR $(95 \% \mathrm{Cl})$ \\
\hline \multicolumn{5}{|l|}{ Age category } \\
\hline Less than 15 years & 15 & 3.36 & 1.00 & 1.00 \\
\hline $15-30$ years & 272 & 60.85 & $1.14(0.62-2.09)$ & $0.82(0.42-1.56)$ \\
\hline $31-45$ years & 100 & 22.37 & $1.09(0.58-2.04)$ & $0.98(0.50-1.93)$ \\
\hline 46 or older & 60 & 13.42 & $1.37(0.7 \mathrm{I}-2.65)$ & $1.40(0.68-2.90)$ \\
\hline \multicolumn{5}{|l|}{ Residence } \\
\hline Urban & 231 & 51.68 & 1.00 & 1.00 \\
\hline Rural & 216 & 48.32 & $0.69(0.57-0.85)$ & $1.12(0.88-1.44)$ \\
\hline \multicolumn{5}{|l|}{ Marital status } \\
\hline Single & 147 & 32.89 & 1.00 & 1.00 \\
\hline Married & 264 & 59.06 & $0.87(0.70-1.08)$ & $0.86(0.68-1.08)$ \\
\hline Divorced & 32 & 7.16 & $2.12(1.42-3.17)$ & $1.35(0.87-2.08)$ \\
\hline Widowed & 4 & 0.89 & $2.45(0.90-6.66)$ & $1.69(0.61-4.66)$ \\
\hline \multicolumn{5}{|l|}{ Educational status } \\
\hline No formal education & 171 & 38.26 & 1.00 & 1.00 \\
\hline Primary & 138 & 30.87 & $1.24(0.98-1.58)$ & $1.18(0.89-1.57)$ \\
\hline Secondary & 103 & 23.04 & $1.68(1.29-2.18)$ & $1.48(1.08-2.03)^{*}$ \\
\hline College and above & 35 & 7.83 & $1.62(1.11-2.38)$ & $1.47(0.92-2.33)$ \\
\hline \multicolumn{5}{|l|}{ Occupation } \\
\hline Farmer/House wife & 187 & 41.83 & 1.00 & 1.00 \\
\hline Employed (gov't/self) & 134 & 29.98 & $2.24(1.74-2.87)$ & $1.36(0.96-1.93)$ \\
\hline Merchant/daily laborer/Student & 126 & 28.19 & $2.39(1.86-3.08)$ & $1.63(1.20-2.22)^{* *}$ \\
\hline \multicolumn{5}{|l|}{ Livelihood setting } \\
\hline Pastoral & 177 & 39.60 & $0.29(0.23-0.37)$ & $0.3 \mathrm{I}(0.24-0.4 \mathrm{I})^{* * * *}$ \\
\hline Non-pastoral & 270 & 60.40 & 1.00 & 1.00 \\
\hline
\end{tabular}

Notes: *p-value $<0.05 ; * *$ P-value $<0.01$; ***p-value $<0.001$.

community where self-prescribing drugs, including antibiotics, are common. In the pastoral community, drugs are available for sale even in open markets and ordinary commodity shops. It is also possible to get potent antibiotics such as free rifampicin which people use as treatment for sexually transmitted infection in such settings. Therefore, those patients who reported not to have any history of previous treatment with anti TB could have such exposure and this can in turn mask the association under discussion.

The other notable result of this study was an association of various drug regimens with the TTSCC. Intensive phase drug regimens containing kanamycin were associated with prolonged time, while regimen containing amikacin was associated with shorter time to the sputum culture conversion. This result is consistent with another in-country study. ${ }^{20}$ These two drugs are from the same family of aminoglycosides, but they may have different effects that need further investigation into why the reported difference existed. Bedaquiline containing regimen was associated with shorter TTSCC except when the regimen includes kanamycin.

Other variables such as older age, sex, diabetes mellitus and history of smoking were reported to be associated with the TTSCC, prolonging the time., ${ }^{9,23,30,31}$ But researchers reported conflicting results on the association of HIV status and history of alcohol abuse with the sputum culture conversion. ${ }^{20,24,27,32}$ In our study, none of these variables were associated with the outcome of interest. This could be due to the difference in study setting as ours mainly involves rural residents than urban dwellers.

\section{Conclusion}

The TTSCC among MDR/RR pulmonary TB patients was longer than two months in the overall study participants and lengthier than three months in patients from the pastoral setting. This is longer than the expected time given pulmonary TB and the ambulatory model of the treatment. The pastoral setting, educational status, undernutrition, previous exposure to anti TB drugs and drug regimen categories were 
Table 2 Association of Clinical Characteristics with the TTSCC Among MDR/RR-TB Patients in Southeastern Oromia, Ethiopia, 202I

\begin{tabular}{|c|c|c|c|c|}
\hline Variables & Number & Percent & COR $(95 \% \mathrm{Cl})$ & COR $(95 \% \mathrm{Cl})$ \\
\hline \multicolumn{5}{|l|}{ Body mass index (BMI) } \\
\hline Less than $18.5 \mathrm{Kg} / \mathrm{SqM}$ & 159 & 37.15 & 1.00 & 1.00 \\
\hline I8.5 or higher $\mathrm{Kg} / \mathrm{SqM}$ & 269 & 62.85 & $1.12(0.91-1.39)$ & $1.35(1.07-1.71)^{*}$ \\
\hline \multicolumn{5}{|l|}{ Resistance type } \\
\hline $\mathrm{RR}$ & 382 & 85.46 & $1.64(1.25-2.15)$ & $1.26(0.89-1.79)$ \\
\hline MDR & 65 & 14.54 & 1.00 & 1.00 \\
\hline \multicolumn{5}{|c|}{ Treatment registration Group } \\
\hline New & 149 & 33.33 & 1.00 & 1.00 \\
\hline Relapse & 119 & 26.62 & $1.07(0.83-1.39)$ & $1.01(0.76-1.33)$ \\
\hline After failure & 179 & 40.04 & $1.88(1.48-2.38)$ & $1.40(1.06-1.84)^{*}$ \\
\hline \multicolumn{5}{|c|}{ Comorbid disease condition } \\
\hline No & 381 & 85.23 & 1.00 & 1.00 \\
\hline Yes & 66 & 14.77 & $0.75(0.57-1.00)$ & $0.91(0.67-1.23)$ \\
\hline \multicolumn{5}{|c|}{ Previously treated with second-line anti TB drug } \\
\hline No & 410 & 91.72 & 1.00 & 1.00 \\
\hline Yes & 37 & 8.28 & $2.38(1.67-3.38)$ & $1.43(0.89-2.28)$ \\
\hline \multicolumn{5}{|c|}{ Intensive phase drug regimen } \\
\hline Cm,Lfx,Cs,Pto(Eto),Z & 188 & 42.06 & 1.00 & 1.00 \\
\hline $\mathrm{Cm}, \mathrm{Lfx}, \mathrm{Eto}, \mathrm{CS}, \mathrm{Z}$ & 173 & 38.70 & $0.38(0.30-0.48)$ & $0.33(0.25-0.45)$ \\
\hline H,E,Z,AM,Mfx,Pto & 13 & 2.91 & $1.33(0.76-2.34)$ & $3.12(1.36-7.14)^{* *}$ \\
\hline $\mathrm{H}, \mathrm{E}, \mathrm{Z}, \mathrm{Km}, \mathrm{Mfx}, \mathrm{Pto}, \mathrm{Cfx}$ & 37 & 8.28 & $0.34(0.23-0.49)$ & $0.55(0.28-1.08)$ \\
\hline Z,Km,Mfx,PAS,Bdq,Cfz & 36 & 8.05 & $0.39(0.23-0.67)$ & $0.23(0.12-0.45)^{* * *}$ \\
\hline \multicolumn{5}{|c|}{ Continuation phase drug regimen } \\
\hline$E, Z, M f x, C f x$ & 50 & 11.19 & 1.00 & 1.00 \\
\hline Lfx,CS,Bdq,Cfx,Lzd & 22 & 4.92 & $2.94(1.70-5.08)$ & $2.48(1.22-5.07)^{*}$ \\
\hline Lfx,CS,Eto,Z,B6 & 368 & 82.33 & $1.29(0.94-1.76)$ & $1.88(0.97-3.64)$ \\
\hline Z,Mfx,PAS,Cfz,LZD & 7 & 1.57 & $2.99(I .34-6.7 I)$ & $6.45(2.45-16.96)^{* * *}$ \\
\hline
\end{tabular}

Notes: *p-value $<0.05$; **P-value $<0.01$; ***p-value $<0.0$

among the significant determinants of the TTSCC among our study participants. Therefore, pastoral community setting requires context TB prevention and control approach.

\section{Abbreviations}

Am, Amikacin; Bdq, Bedaquiline; Cm, Capreomycin; Cfz, Clofazimine; Cs, Cycloserine; E, Ethambutol; Eto, Ethionamide; Km, Kanamycin; Lfx, Levofloxacin; Lzd, Linezolid; Mfx, Moxifloxacin; PAS, Para-aminosalicylic acid; Pto, Protionamide; Z, Pyrazinamide.

\section{Data Sharing Statement}

Data used for this study can be accessed by a formal request from the paper's corresponding author.

\section{Ethics Approval and Informed Consent}

Ethical clearance was obtained from Addis Ababa University Ethical Review board. Upon the ethical approval, a permission letter to conduct the study was obtained from Oromia regional health bureau and submitted to the selected hospitals. Tuberculosis clinic heads gave consent for accessing and extracting data from records at each hospital. The consent included consent for publication. Patient names and identification numbers were removed to ensure the confidentiality of patient information. Instead, anonymous codes were used to identify individual records for data cleaning. In general, this study was conducted in accordance with the Helsinki declaration.

\section{Acknowledgments}

The authors would like to thank Addis Ababa University, College of Medicine and Health Science, for providing financial support for the process of data collection. The authors would also like to thank the selected health facilities leaders and the data collectors for their support, without which this work would not have been practical. 


\section{Author Contributions}

All authors made a significant contribution to the work reported, whether that is in the conception, study design, execution, acquisition of data, analysis and interpretation, or in all these areas; took part in drafting, revising or critically reviewing the article; gave final approval of the version to be published; have agreed on the journal to which the article has been submitted; and agree to be accountable for all aspects of the work.

\section{Funding}

No funding was obtained from any funding agency. It was conducted using a small grant provided by Addis Ababa University to cover costs spent for data collection purposes.

\section{Disclosure}

All authors of this paper confirm that they have no conflicts of interest in this study.

\section{References}

1. World Health Organization. Guidance for the surveillance of drug resistance in tuberculosis. World Health Organization; 2020.

2. World Health Organization. Global tuberculosis report 2020. World Health Organization; 2020.

3. Federal Ministry of Health Ethiopia. Guidelines for clinical and programmatic management of TB, TB/ HIV,DR-TB and leprosy in Ethiopia; 2021.

4. Ki-moon B. Building a tuberculosis-free world on a foundation of universal health coverage. Lancet. 2019;393:1268-1270. doi:10.1016/S0140-6736(19)30433-7

5. World Health Organization. End TB strategy. Global strategy and targets for tuberculosis prevention, care and control after 2015. Geneva; 2014.

6. Federal Ministry of Health Ethiopia. Guidelines for TB, DR-TB and LEPROSY in Ethiopia, sixth edition; 2017.

7. Kebede A, Beyene D, Yenew B, et al. Monitoring quality indicators for the Xpert MTB/RIF molecular assay in Ethiopia. PLoS One. 2019;14(11):e0225205. doi:10.1371/journal.pone.0225205

8. World Health Organization. WHO consolidated guidelines on drug-resistant tuberculosis treatment; 2019.

9. Liu Q, Lu P, Martinez L, et al. Factors affecting time to sputum culture conversion and treatment outcome of patients with multidrug-resistant tuberculosis in China. BMC Infect Dis. 2018;18:114. doi:10.1186/s12879-018-3021-0

10. Russkikh A, Korotych O, Sereda Y, et al. Factors associated with culture conversion among adults treated for pulmonary extensively drug-resistant tuberculosis during 2018-2019 in the Russian Federation: an observational cohort study. Monaldi Arch Chest Dis. 2021;91. doi:10.4081/monaldi.2021.1678

11. Velayutham B, Nair D, Kannan T, et al. Factors associated with sputum culture conversion in multi-drug- resistant pulmonary tuberculosis. Int $J$ Tuberc Lung Dis. 2016;20:1671-1676. doi:10.5588/ijtld.16.0096

12. Diktanas S, Korotych O, Sereda Y, et al. Factors associated with time to sputum culture conversion of rifampicin-resistant tuberculosis patients in Klaipeda, Lithuania in 2016-2019: a cohort study. Monaldi Arch Chest Dis. 2021;91. doi:10.4081/monaldi.2021.1675
13. Kapata N, Chanda-Kapata P, Michelo C. The social determinants of tuberculosis and their association with TB-HIV co-infection in Lusaka, Zambia. Med J Zambia. 2013;40:7.

14. Hargreaves JR, Boccia D, Evans CA, et al. The social determinants of tuberculosis: from evidence to action. Am $J$ Public Health. 2011;101:654-662. doi:10.2105/AJPH.2010.199505

15. Duarte R, Lönnroth K, Carvalho C, et al. Tuberculosis, social determinants and co-morbidities (including HIV). Pulmonology. 2018;24:115-119. doi:10.1016/j.rppnen.2017.11.003

16. Megerso A, Deyessa N, Jarso G, Tezera R, Worku A. Exploring community tuberculosis program in the pastoralist setting of Ethiopia: a qualitative study of community health workers' perspectives in Borena Zone, Oromia Region. BMC Health Serv Res. 2021;21:632. doi:10.1186/s12913-021-06683-y

17. Megerso A, Deyessa N, Jarso G, Worku A. Lived experiences of tuberculosis patients and their implications for early tuberculosis case identification and management in pastoralist community setting: a qualitative study in Borena zone, Oromia region of Ethiopia. BMC Health Serv Res. 2020;20:933. doi:10.1186/s12913-02005787-1

18. Dupont WD, Plummer WD. Power and sample size calculations: a review and computer program. Control Clin Trials. 1990;11 (2):116-128. doi:10.1016/0197-2456(90)90005-M

19. Halabi S, Singh B. Sample size determination for comparing several survival curves with unequal allocations. Stat Med. 2004;23:1793-1815. doi:10.1002/sim.1771

20. Shibabaw A, Gelaw B, Wang S-H, Tessema B. Time to sputum smear and culture conversions in multi-drug resistant tuberculosis at University of Gondar Hospital, Northwest Ethiopia. PLoS One. 2018;13:e0198080. doi:10.1371/journal.pone. 0198080

21. IBM SPSS Statistics. IBM SPSS statistics for windows, version 26.0. Armonk, NY: IBM Corp.; 2019.

22. Center for Disease Control. Epi info ${ }^{\mathrm{TM}} 7$ user guide for version 7.2. 645; 2016.

23. Magee MJ, Kempker RR, Kipiani M, et al. Diabetes mellitus, smoking status, and rate of sputum culture conversion in patients with multidrug-resistant tuberculosis: a cohort study from the country of Georgia. PLoS One. 2014;9:e94890. doi:10.1371/journal.pone.00 94890

24. Yihunie Akalu T, Muchie KF, Alemu Gelaye K, Munderloh UG. Time to sputum culture conversion and its determinants among multi-drug resistant tuberculosis patients at public hospitals of the Amhara Regional State: a multicenter retrospective follow up study. PLoS One. 2018;13:e0199320. doi:10.1371/journal.pone.0199320

25. Martinson NA, Ncha R, Variava E, Otwombe K, Kawonga M. Predictors of time to sputum culture conversion in multi-drugresistant tuberculosis and extensively drug-resistant tuberculosis in patients at Tshepong-Klerksdorp Hospital. South Afr J Infect Dis. 2019;34:1-8.

26. Tekalegn Y, Woldeyohannes D, Assefa T, Aman R, Sahiledengle B. Predictors of time to sputum culture conversion among drug-resistant tuberculosis patients in Oromia region hospitals, Ethiopia. Infect Drug Resist. 2020;13:2547-2556. doi:10.2147/ IDR.S250878

27. Tierney DB, Franke MF, Becerra MC, et al. Time to culture conversion and regimen composition in multidrug-resistant tuberculosis treatment. PLoS One. 2014;9:e108035. doi:10.1371/journal.pone.0108035

28. Assemie MA, Alene M, Petrucka P, Leshargie CT, Ketema DB. Time to sputum culture conversion and its associated factors among multidrug-resistant tuberculosis patients in Eastern Africa: a systematic review and meta-analysis. Int $J$ Infect Dis. 2020;98:230-236. doi:10.1016/j.ijid.2020.06.029

29. Park H-O, Kim SH, Moon SH, et al. Association between body mass index and sputum culture conversion among South Korean patients with multidrug resistant tuberculosis in a tuberculosis referral hospital. Infect Chemother. 2016;48:317. doi:10.3947/ic.2016.48.4.317 
30. Putri FA, Burhan E, Nawas A, et al. Body mass index predictive of sputum culture conversion among MDR-TB patients in Indonesia. Int J Tuberculosis Lung Dis. 2014;18(5):564-570. doi:10.5588/ ijtld.13.0602

31. Salindri AD, Kipiani M, Kempker RR, et al. Diabetes reduces the rate of sputum culture conversion in patients with newly diagnosed multidrug-resistant tuberculosis. Open Forum Infect Dis. 2016;3: ofw126. doi:10.1093/ofid/ofw126
32. Lu P, Liu Q, Martinez L, et al. Time to sputum culture conversion and treatment outcome of patients with multidrug-resistant tuberculosis: a prospective cohort study from urban China. Eur Respir J. 2017;49:1601558. doi:10.1183/13993003.01558-2016

\section{Publish your work in this journal}

Infection and Drug Resistance is an international, peer-reviewed openaccess journal that focuses on the optimal treatment of infection (bacterial, fungal and viral) and the development and institution of preventive strategies to minimize the development and spread of resistance. The journal is specifically concerned with the epidemiology of antibiotic resistance and the mechanisms of resistance development and diffusion in both hospitals and the community. The manuscript management system is completely online and includes a very quick and fair peerreview system, which is all easy to use. Visit http://www.dovepress.com/ testimonials.php to read real quotes from published authors. 